\title{
Keynote Abstract: Machine Learning in Conflict Studies: Reflections on Ethics, Collaboration, and Ongoing Challenges
}

\author{
Kristine Eck \\ Uppsala University \\ Uppsala, Sweden \\ kristine.eckepcr.uu.se
}

Advances in machine learning are nothing short of revolutionary in their potential to analyze massive amounts of data and in doing so, create new knowledge bases. But there is a responsibility in wielding the power to analyze these data since the public attributes a high degree of confidence to results which are based on big datasets.

In this keynote, I will first address our ethical imperative as scholars to "get it right." This imperative relates not only to model precision but also to the quality of the underlying data, and to whether the models inadvertently reproduce or obscure political biases in the source material. In considering the ethical imperative to get it right, it is also important to define what is "right": what is considered an acceptable threshold for classification success needs to be understood in light of the project's objectives.

I then reflect on the different topics and data which are sourced in this field. Much of the existing research has focused on identifying conflict events (e.g. battles), but scholars are also increasingly turning to ML approaches to address other facets of the conflict environment.

Conflict event extraction has long been a challenge for the natural language processing (NLP) community because it requires sophisticated methods for defining event ontologies, creating language resources, and developing algorithmic approaches. NLP machine-learning tools are ill-adapted to the complex, often messy, and diverse data generated during conflicts. Relative to other types of NLP text corpora, conflicts tend to generate less textual data, and texts are generated non-systematically. Conflict-related texts are often lexically idiosyncratic and tend to be written differently across actors, periods, and conflicts. Event definition and adjudication present tough challenges in the context of conflict corpora.
Topics which rely on other types of data may be better-suited to NLP and machine learning methods. For example, Twitter and other social media data lend themselves well to studying hate speech, public opinion, social polarization, or discursive aspects of conflictual environments. Likewise, government-produced policy documents have typically been analyzed with historical, qualitative methods but their standardized formats and quantity suggest that ML methods can provide new traction. ML approaches may also allow scholars to exploit local sources and multi-language sources to a greater degree than has been possible.

Many challenges remain, and these are best addressed in collaborative projects which build on interdisciplinary expertise. Classification projects need to be anchored in the theoretical interests of scholars of political violence if the data they produce are to be put to analytical use. There are few ontologies for classification that adequately reflect conflict researchers' interests, which highlights the need for conceptual as well as technical development.

Bio: Kristine Eck is an Associate Professor at the Department of Peace and Conflict Research at Uppsala University, where she serves as the Director of the Uppsala Rotary Peace Center. Her research interests concern coercion and resistance, including human rights, police misconduct, state surveillance, and conflict data production. She served as the Director of the Uppsala Conflict Data Program (UCDP) 2017-2018 and has been a Visiting Researcher at Oxford University, Copenhagen University, the University of Notre Dame, and Kobe University. Dr. Eck's research has been funded by the Swedish Research Council, the Swedish Foundation for Humanities and Social Sciences, and the Norwegian Foreign Ministry. 